\title{
Anomalias congênitas do trato urinário superior: novas imagens das mesmas doenças*
}

\author{
Congenital upper urinary tract abnormalities: new images of the same diseases \\ Carol Pontes de Miranda Maranhão ${ }^{1}$, Christiana Maia Nobre Rocha de Miranda ${ }^{2}$, Carla Jotta
Justo dos Santos ${ }^{1}$, Lucas de Pádua Gomes de Farias ${ }^{3}$, Igor Gomes Padilha ${ }^{3}$
}

Resumo As anomalias congênitas do trato urinário superior implicam modificações morfofuncionais com espectro clínico variável, desde manifestações assintomáticas até falência renal e incompatibilidade com a vida. A tomografia computadorizada, além de ter superado o método de imagem da urografia excretora, tem desempenhado papel fundamental no diagnóstico das anomalias congênitas, orientando melhor nas decisões terapêuticas clínicas e cirúrgicas, além de atuar como ferramenta essencial na identificação de complicações associadas e no melhor desempenho de técnicas operatórias menos invasivas.

Unitermos: Anomalias congênitas; Trato urinário; Tomografia computadorizada multidetectores.

Abstract Congenital upper urinary tract abnormalities imply a variable clinical spectrum of morphofunctional changes ranging from asymptomatic conditions to renal failure and incompatibility with life. Computed tomography, which has overcome excretory urography imaging, has been playing a key role in the diagnosis of congenital anomalies, serving as a better guidance in the therapeutic and surgical decision-making process, besides acting as an essential tool in the identification of associated complications and aiding in the performance of minimally invasive surgery techniques.

Keywords: Congenital abnormalities; Urinary tract; Multislice computed tomography.

Maranhão CPM, Miranda CMNR, Santos CJJ, Farias LPG, Padilha IG. Anomalias congênitas do trato urinário superior: novas imagens das mesmas doenças. Radiol Bras. 2013 Jan/Fev;46(1):43-50.

\section{INTRODUÇÃO}

Embriologicamente, os sistemas urinário e genital estão bastante relacionados, uma vez que o cordão nefrogênico e a crista gonadal surgem de uma elevação longitudinal do mesoderma em cada lado da aorta dorsal $^{(\mathbf{1})}$. Aproximadamente $10 \%$ de todas as pessoas nascem com malformações potencialmente significativas do sistema urinário ${ }^{(2)}$, sendo as alterações estruturais e funcionais do desenvolvimento responsáveis por $20 \%$ a $30 \%$ das anomalias identificadas no período pré-natal, o que consti-

* Trabalho realizado na Clínica de Medicina Nuclear e Radiologia de Maceió (MedRadiUS), Maceió, AL, Brasil.

1. Membros Titulares do Colégio Brasileiro de Radiologia e Diagnóstico por Imagem (CBR), Médicas da Clínica de Medicina Nuclear e Radiologia de Maceió (MedRadiUS), Maceió, AL, Brasil.

2. Doutora, Médica da Clínica de Medicina Nuclear e Radiologia de Maceió (MedRadiUS), Professora de Radiologia e Diag nóstico por Imagem da Universidade Federal de Alagoas (UFAL), Maceió, AL, Brasil.

3. Estudantes do $6^{\circ}$ ano da Faculdade de Medicina da Universidade Federal de Alagoas (UFAL), Maceió, AL, Brasil.

Endereço para correspondência: Dra. Christiana Maia Nobre Rocha de Miranda. Rua Hugo Corrêa Paes, 104, Farol. Maceió, AL, Brasil, 57050-730. E-mail: maiachristiana@globo.com.

Recebido para publicação em 16/6/2012. Aceito, após revisão, em 8/10/2012. tui uma das principais causas de falência renal em crianças ${ }^{(3-5)}$.

As anomalias congênitas do trato urinário superior, incluindo-se as formas mais brandas, não são $\operatorname{raras}^{(5)}$. Em 3\% a 4\% dos recém-nascidos ocorre alguma anormalidade dos rins e dos ureteres, sendo as anomalias da forma e posição dos rins as mais comuns $^{(\mathbf{1})}$. A maior parte dessas doenças é apenas acompanhada clinicamente, fazendo-se necessário um correto diagnóstico da alteração morfológica, assim como a correta avaliação de possíveis complicações.

Anteriormente, as radiografias simples do abdome e a urografia excretora (UE) eram os métodos de escolha no diagnóstico por imagem dos rins e das vias urinárias. Porém, a introdução da tomografia computadorizada (TC) e da ressonância magnética (RM) influenciou bastante a utilização dos métodos de imagem no diagnóstico e tratamento. Desde a década passada, a TC tem ultrapassado a UE na avaliação do trato geniturinário $^{(6-8)}$.

Relata-se que a TC é superior em relação à UE e à ultrassonografia (US) na detecção e caracterização das massas re- nais ${ }^{(\mathbf{9 , 1 0})}$, assim como, recentemente, sua superioridade em detectar litíase do trato urinário $^{(\mathbf{8 , 1 1 , 1 2})}$. A única potencial limitação da TC, a limitada acurácia na avaliação da superfície mucosa do sistema coletor renal e dos ureteres, tem sido superada com os recentes avanços da tomografia computadorizada com multidetectores (TCMD), realizando cortes cada vez mais finos em um curto período de tempo, o que implica uma maior dose de radiação a ser utilizada. As reconstruções multiplanares e as imagens pós-processamento dos aparelhos de TCMD permitem diagnósticos cada vez mais precisos $^{(\mathbf{6}, \mathbf{1 3})}$.

O objetivo deste artigo é demonstrar, por meio da TCMD, as principais anomalias congênitas renais que podem ser detectadas em pacientes de ambos os sexos e faixa etária variadas por meio dos planos anatômicos convencionais e técnicas de renderização de volume (VRT).

\section{ANOMALIAS DO TAMANHO}

As anomalias do tamanho, da forma e da posição dos rins ocorrem nas fases ini- 
ciais do desenvolvimento e são resultantes da incorreta união entre os blastemas metanéfricos ${ }^{(\mathbf{1})}$.

No rim hipoplásico há falência do desenvolvimento, e apesar de pequenos e pouco numerosos, os componentes do sistema coletor funcionam normalmente e guardam relação com o volume do parênquima, características que devem ser diferenciadas do rim atrófico adquirido, pequeno e contraído. A presença de hipoplasia renal tem sido associada a infecções e hipertensão $\operatorname{arterial}^{(\mathbf{1 4 , 1 5})}$.

A hiperplasia, outra anomalia do tamanho, é associada à agenesia ou hipoplasia do lado contralateral, sendo denominado, de forma mais adequada, hipertrofia compensatória $^{(16,17)}$.

\section{ANOMALIAS DA FORMA}

Durante a migração dos rins para a fossa renal, eles cruzam as artérias umbilicais. Qualquer alteração na posição destas artérias pode causar a fusão dos blastemas nefrogênicos ${ }^{(\mathbf{1 8})}$, resultando em uma fusão que pode ser parcial, como ocorre nos rins em ferradura e na ectopia renal cruzada com fusão, ou total, como no rim em panqueca $^{(19,20)}$.

O rim em ferradura (Figuras 1A e 1B) é a anomalia renal mais comum e mais encontrada entre os homens. O grau de fusão renal é variável e ocorre, na maioria dos casos, entre os polos inferiores dos rins, que se encontram mais próximos da linha média do que os rins normais. $\mathrm{O}$ istmo, situado mais comumente na frente da aorta ou da veia cava inferior, une as duas massas renais e pode conter parênquima funcionante ou corresponder a uma faixa de tecido fibroso, por isso pode ser necessária a sua avaliação funcional por meio de radionuclídeo antes de qualquer procedimento intervencionista. $\mathrm{O}$ próprio istmo dificulta a rotação renal, assim como sua ascensão devido à artéria mesentérica inferior. Seu suprimento sanguíneo pode ser variado e, usualmente, o sistema coletor encontra-se anteriorizado ${ }^{(\mathbf{1 , 1 8 - 2 1 )}}$.

A maioria dos pacientes é assintomática e o achado é incidental durante exames de imagem. Quando sintomáticos, geralmente são relatados hidronefrose (Figura 2), infecção ou formação calculosa. Tem sido
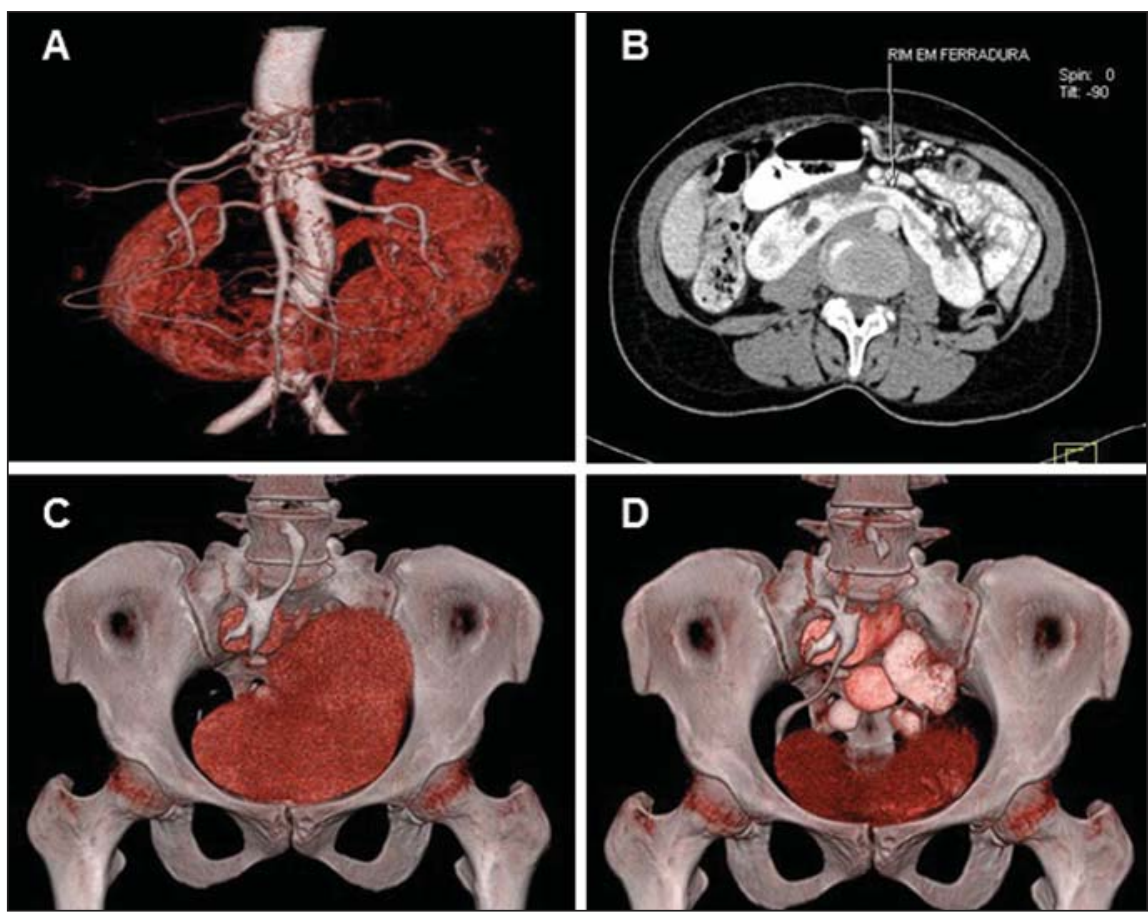

Figura 1. Anomalias da forma renal. Imagens de TCMD em VRT (A) e axial (B) do rim em ferradura mostrando a união renal pelos polos inferiores. Notar a captação de contraste pelo istmo e a sua posição anterior em relação à artéria aorta, próximo à bifurcação das artérias ilíacas comuns. Imagens de TCMD em VRT (C,D) do rim em panqueca.

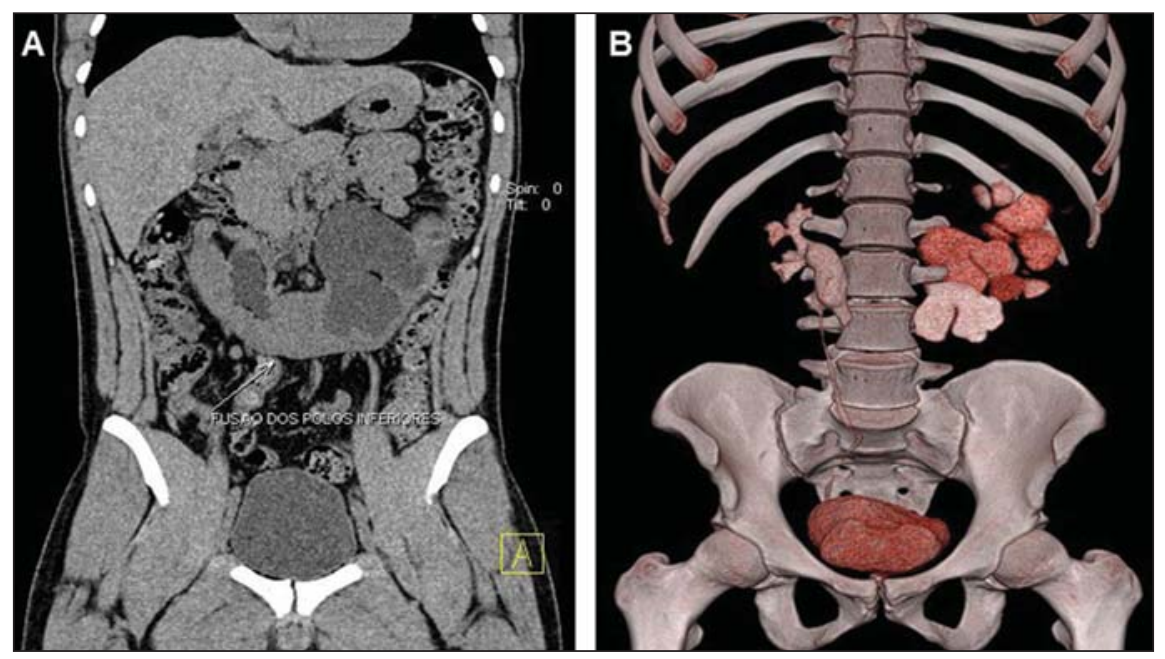

Figura 2. Imagens de TCMD do rim em ferradura. Imagens coronal (A) e em VRT (B) identificando a união renal pelos polos inferiores e a presença de hidronefrose à esquerda.

associada a uma maior propensão para neoplasias malignas, como o tumor de Wilms, assim como malformações sistêmicas, como na síndrome de Turner ${ }^{(\mathbf{1}, \mathbf{1 8 , 2 0})}$.

$\mathrm{Na}$ anomalia que caracteriza os rins em panqueca (Figuras 1C e 1D), os rins formam uma massa única, mediana, situada na cavidade pélvica ou ao nível da bifurcação aórtica, de aspecto achatado, não reniforme, lobulado, com o sistema coletor anteriori- zado e ureteres curtos, que drenam em orifícios independentes ou em ureter único ${ }^{(20)}$. Sua irrigação sanguínea, também anômala, é fator de risco para o comprimento vascular renal, desde uma simples gestação até traumas pélvicos ${ }^{(\mathbf{1 9})}$.

De tal forma, a identificação das anomalias de fusão renal não consiste em pior prognóstico, porém devem ser precocemente identificadas para a avaliação de 
afecções concomitantes, assim como para os diagnósticos diferenciais que simulam massa pélvica e que não poderão ser inequivocamente removidas ou lesionadas ${ }^{(19)}$

\section{ANOMALIAS DA POSIÇÃO}

O rim ectópico (Figuras 3 e 4) resulta da falha na migração deste da cavidade pélvica para a fossa renal ${ }^{(15)}$ e comumente está relacionado a má rotação ${ }^{(\mathbf{1 4}, 18)}$. Observa-se leve predileção pelo lado esquerdo e $10 \%$ dos casos podem ser bilaterais ${ }^{(\mathbf{1 8})}$.

A ectopia cranial é usualmente intratorácica e a ectopia caudal pode ser classificada em abdominal, ilíaca (lombar) ou pél- vica (sacral), sendo esta a mais comum ${ }^{(\mathbf{1 5})}$, além da associação com malformações genitais $^{(14)}$.

Na ectopia renal congênita, a posição mais baixa do rim é acompanhada de um ureter mais curto, de vasos renais com origem ectópica (vasos sanguíneos próximos a ele, podendo ser irrigado por vasos múltiplos) e algum grau de malformação do sistema coletor ${ }^{(\mathbf{1 , 1 8})}$ que torna esta condição mais suscetível a refluxo, infecções, litíase e quadros obstrutivos ${ }^{(2)}$. Não deve ser confundido com o rim anormalmente móvel, nem com o rim ptótico ${ }^{(\mathbf{1 8})}$.

Na ectopia simples (Figura 3), o rim está do mesmo lado em que se originou. A lo-
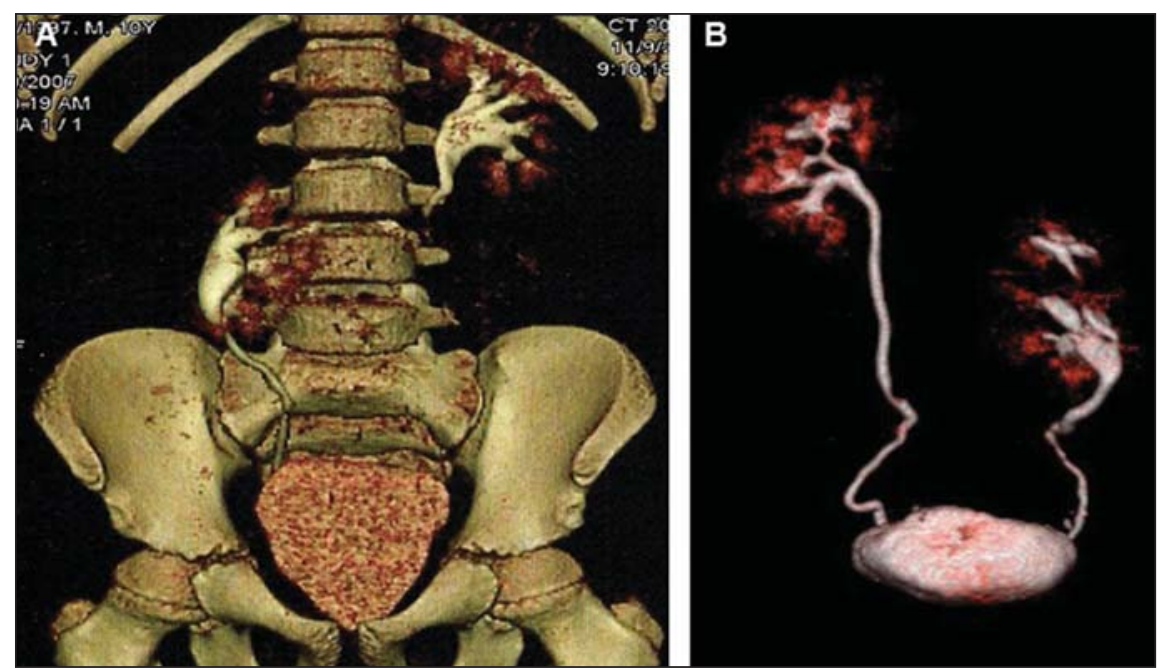

Figura 3. Imagens de TCMD em VRT da ectopia renal simples. Evidencia-se a associação com anomalias de rotação à direita $(\mathbf{A})$ e à esquerda $(\mathbf{B})$. calização pélvica, a mais comum, está associada a ausência de sua morfologia habitual, pois o rim é frequentemente mal rotacionado e tem sua imagem superposta aos ossos da bacia, dificultando a sua identificação ${ }^{(\mathbf{1 8})}$.

A ectopia renal cruzada (Figura 4) se dá quando um dos rins se encontra contralateral à inserção de seu ureter na bexiga urinária. $\mathrm{O}$ rim ectópico quase sempre tem ureter mais curto, e, portanto, situação mais baixa em relação ao rim normal, que pode exibir grau variado de ptose e de vício de rotação. O rim normal pode manter-se separado do rim ectópico ou formar massa única com ele ${ }^{(\mathbf{1 4 , 1 5})}$. Quando em fusão, $85 \%$ dos casos, pode ser identificada em diversas formas de apresentação, sendo mais comum a fusão do polo superior do rim ectópico com o polo inferior do outro ${ }^{(\mathbf{1 8 , 2 0})}$.

\section{ANOMALIAS DE ROTAÇÃO}

A migração do rim, desde a cavidade pélvica até o seu sítio lombar definitivo, ocorre simultaneamente com a sua rotação no plano longitudinal. À medida que migra cefalicamente, cada rim sofre uma rotação medial de aproximadamente $90^{\circ}$, e os hilos se orientam para a linha média, alinhados e voltados um para o outro anteromedialmente ${ }^{(\mathbf{1 , 1 8})}$. É importante estabelecer um correto diagnóstico a fim de excluir outras condições patológicas que podem produzir distorção similar dos rins ${ }^{(\mathbf{1 8})}$.

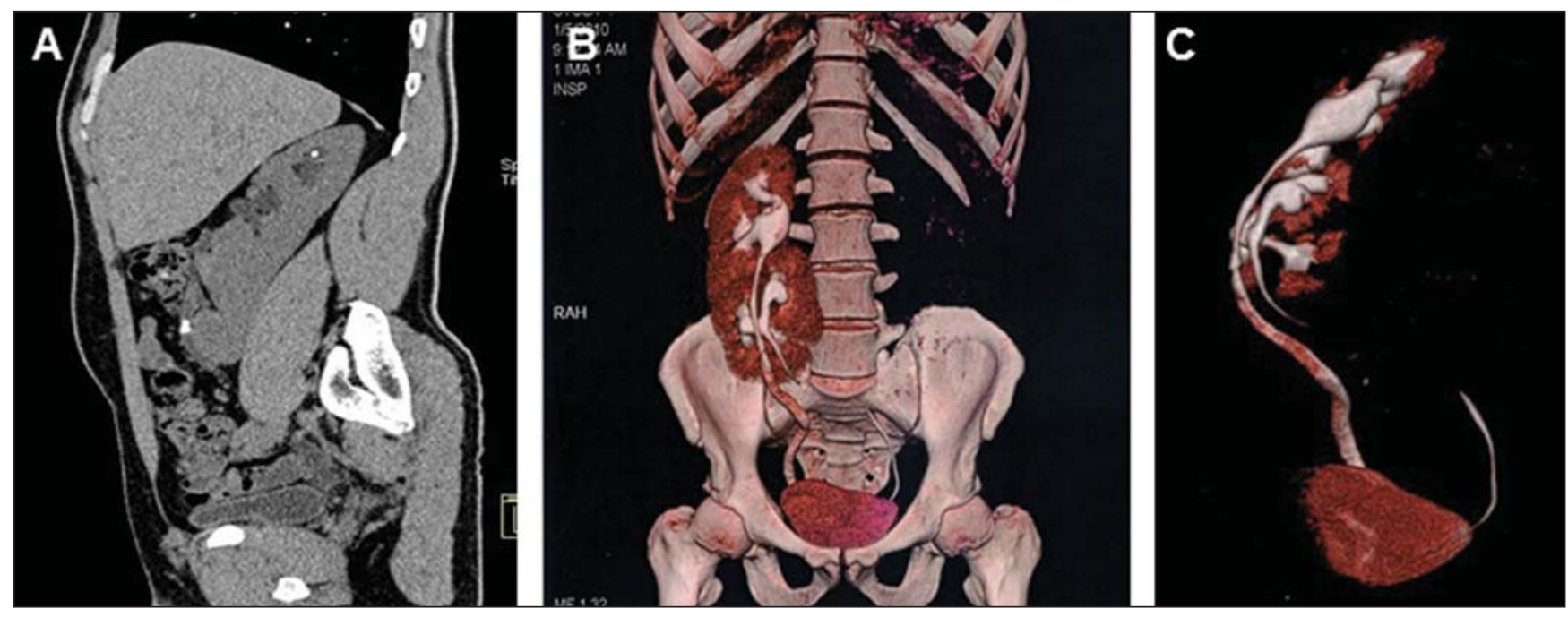

Figura 4. Imagens de TCMD da ectopia renal cruzada. Imagens oblíqua $(\mathbf{A})$ e em VRT (B,C) demonstrando duplicidade renal à direita sem a fusão de massas renais. Notar que o ureter do rim superior tem seu trajeto à direita, enquanto o ureter do rim inferior, ectópico, cruza para o lado contralateral e insere-se na bexiga urinária. 
A má rotação dos rins (Figura 5) é comumente associada a um rim ectópico ou em fusão, além da possibilidade de obstrução parcial da pelve e ureter dos rins. Pode ocorre em ambos os rins e observam-se mais a rotação incompleta e a não rotação quando comparada aos outros subtipos ${ }^{(\mathbf{1 8})}$. Raramente ocorre uma superrotação dispondo o hilo renal voltado para o dorso ${ }^{(15)}$.

\section{ANOMALIAS DE NÚMERO}

A agenesia renal, com provável causa multifatorial, é definida como ausência de tecido renal secundária a falha na embriogênese, podendo ser uni ou bilateral ${ }^{(\mathbf{1})}$. $\mathrm{O}$ rim supranumerário é extremamente raro e acha-se separado dos outros dois, além de possuir suprimento sanguíneo próprio ${ }^{(\mathbf{1 4})}$.
A agenesia unilateral (Figura 6) é uma anomalia relativamente comum que ocorre aproximadamente $1 \mathrm{vez}$ em cada 1.000 recém-nascidos ${ }^{(\mathbf{1})}$. Possui bom prognóstico quando não associada a outras anomalias sistêmicas e está relacionada ao rim contralateral geralmente hipertrofiado como resultado compensatório ${ }^{(\mathbf{1 , 2})}$. A agenesia da glândula adrenal ipsilateral é encontrada

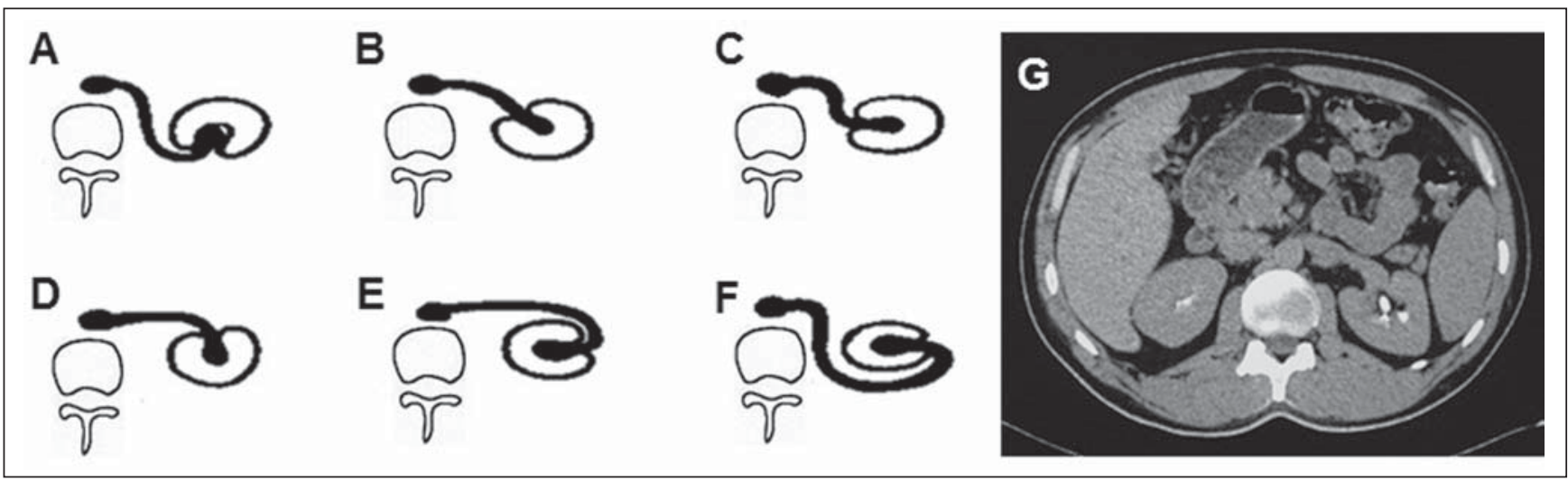

Figura 5. Anomalias de rotação renal. Ilustração esquemática do rim primitivo fetal (A), rim normal do adulto (B), rotação incompleta (C), hiper-rotação (D), hiperrotação exagerada $(\mathbf{E})$ e rotação invertida $(\mathbf{F})$. (Adaptado de Prando et al. ${ }^{(\mathbf{5})}$ ). Imagem de TCMD $(\mathbf{G})$ em corte axial evidenciando hiper-rotação renal à esquerda.

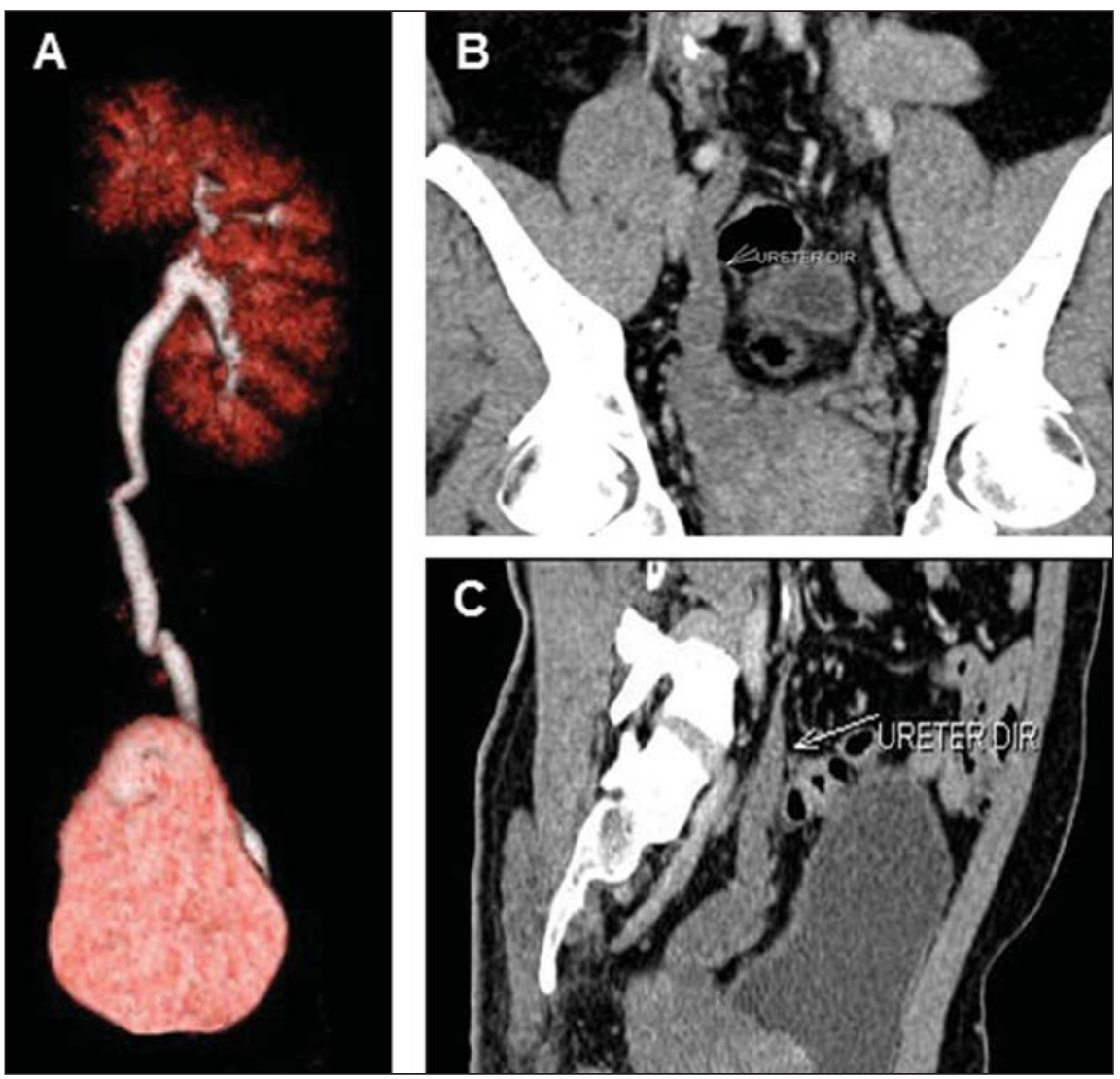

Figura 6. Agenesia renal unilateral. Imagem de TCMD em VRT (A) mostrando rim esquerdo único. Notar, nas imagens de TCMD coronal $(\mathbf{B})$ e sagital $(\mathbf{C})$, que o ureter direito é rudimentar e desemboca ectopicamente na vesícula seminal direita. em $10 \%$ dos $\operatorname{casos}^{(22)}$ e a artéria e a veia renais não se desenvolvem. O ureter correspondente está ausente na maioria dos casos, podendo corresponder a um cordão fibroso que pode terminar ectopicamente, por exemplo, na vesícula seminal contralateral $^{(15)}$. Deve-se suspeitar de agenesia renal unilateral em crianças com somente uma artéria umbilical ${ }^{(\mathbf{1})}$.

A agenesia bilateral ocorre 1 vez em cada 3.000 recém-nascidos, sendo incompatível com a vida e geralmente encontrada em natimortos $^{(\mathbf{1 , 2})}$. Estas crianças têm um aspecto facial característico ${ }^{(\mathbf{1 , 2 3})}$ e estão frequentemente associadas a outros transtornos congênitos, como ocorre na síndrome de Potter ${ }^{(2,24)}$. A urina fetal não é produzida, resultando em grave oligodrâmnia ${ }^{(24)}$.

\section{ANOMALIAS DA ANATOMIA LOBAR}

Os defeitos da anatomia cortical dos rins, como a persistência das lobulações fetais, "corcova de dromedário" e hipertrofia dos septos de Bertin, representam variações anatômicas frequentes do parênquima renal e podem simular tumores renais, porém com o parênquima saudável ${ }^{(21,23)}$ (Figura 7). 


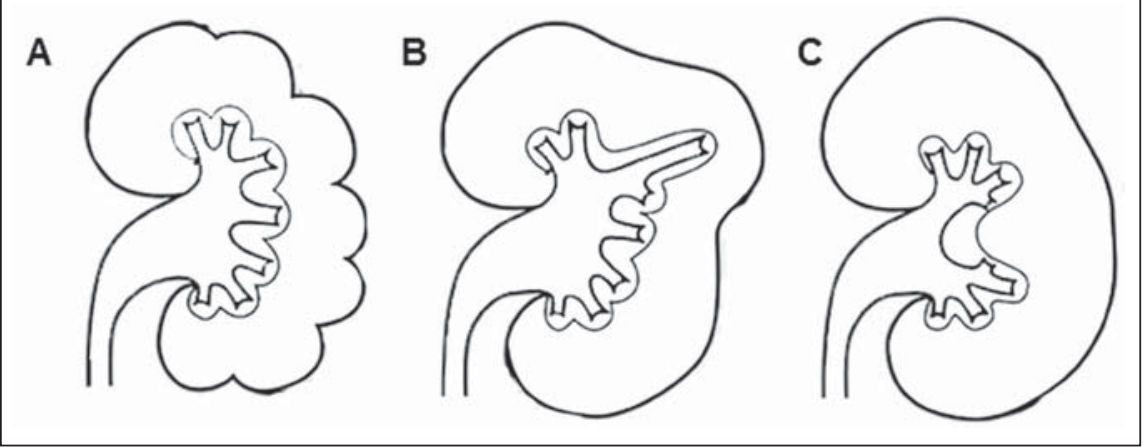

Figura 7. Anomalias da anatomia lobar. Ilustração esquemática da persistência das lobulações fetais (A), do rim em corcova de dromedário (B) e da hipertrofia dos septos de Bertin (C). (Adaptado de Dyer et al. $\left.{ }^{(21)}\right)$.

As lobulações do contorno (Figura 7A) renal são vistas em cerca de 5\% dos adultos submetidos ao estudo por imagem dos rins ${ }^{(\mathbf{2 3})}$. Trata-se da persistência de sulcos corticais bem definidos na superfície renal que são encontrados no rim fetal e costumam desaparecer durante a infância como consequência do crescimento e aumento do número de néfrons ${ }^{(\mathbf{1})}$. Podem ser ainda confundidos com cicatrizes renais ${ }^{(\mathbf{2 3 , 2 5})}$.

Já o rim em "corcova de dromedário" (Figura 7B) caracteriza-se por ser uma alteração da forma e do contorno da face posterolateral do rim esquerdo consequente a uma proeminência focal do parênquima renal, provavelmente devido a uma impressão do baço durante a vida embrionária ${ }^{(23,25)}$.

Outra condição benigna que pode simular neoplasia é a hipertrofia do septo de Bertin (Figuras 7C e 8), que corresponde a colunas de tecido cortical renal, localizadas entre as pirâmides, resultantes da fusão de dois ou mais lobos renais. Essas colunas podem ser mais espessas, hipertróficas e profundas, protruindo no seio renal e manifestando-se como nódulo cortical de aspecto regular, bem definido, situado na junção do terço renal superior com o médio ${ }^{(\mathbf{2 3 , 2 6})}$.

A hipertrofia do septo de Bertin possui sinais que são sugestivos à UE e à US, porém não específicos, devendo-se realizar $\mathrm{TC}^{(25)}$, cujos achados são bem característicos, apresentando-se isodensos em relação ao parênquima cortical e impregnando-se uniformemente pós-contraste.

\section{ANOMALIAS DOS CÁLICES E DAS PAPILAS}

O divertículo pielocalicinal é uma cavidade cística, revestida de urotélio, situada no interior do parênquima renal e que pode ser adquirida ou congênita (mais comum) e única ou múltipla ${ }^{(2,15)}$. Podem ser divididos em dois tipos: 1) o mais frequente é representado por lesões menores que acometem os cálices menores e que estão próximas à região do polo renal superior (Figura 9); 2) o menos frequente, e de localização central nos rins, está relacionado a pelve renal ou cálices maiores ${ }^{(27,28)}$

Os divertículos menores são normalmente assintomáticos e achados incidentais aos exames de imagem. Os maiores geralmente são sintomáticos e a estase urinária favorece o surgimento de infecção urinária e a formação de cálculos ${ }^{(2,27)}$. Enquanto a incidência do divertículo pielocalicinal é baixa, a frequência da formação calculosa associada ao divertículo é alta ${ }^{(29)}$.
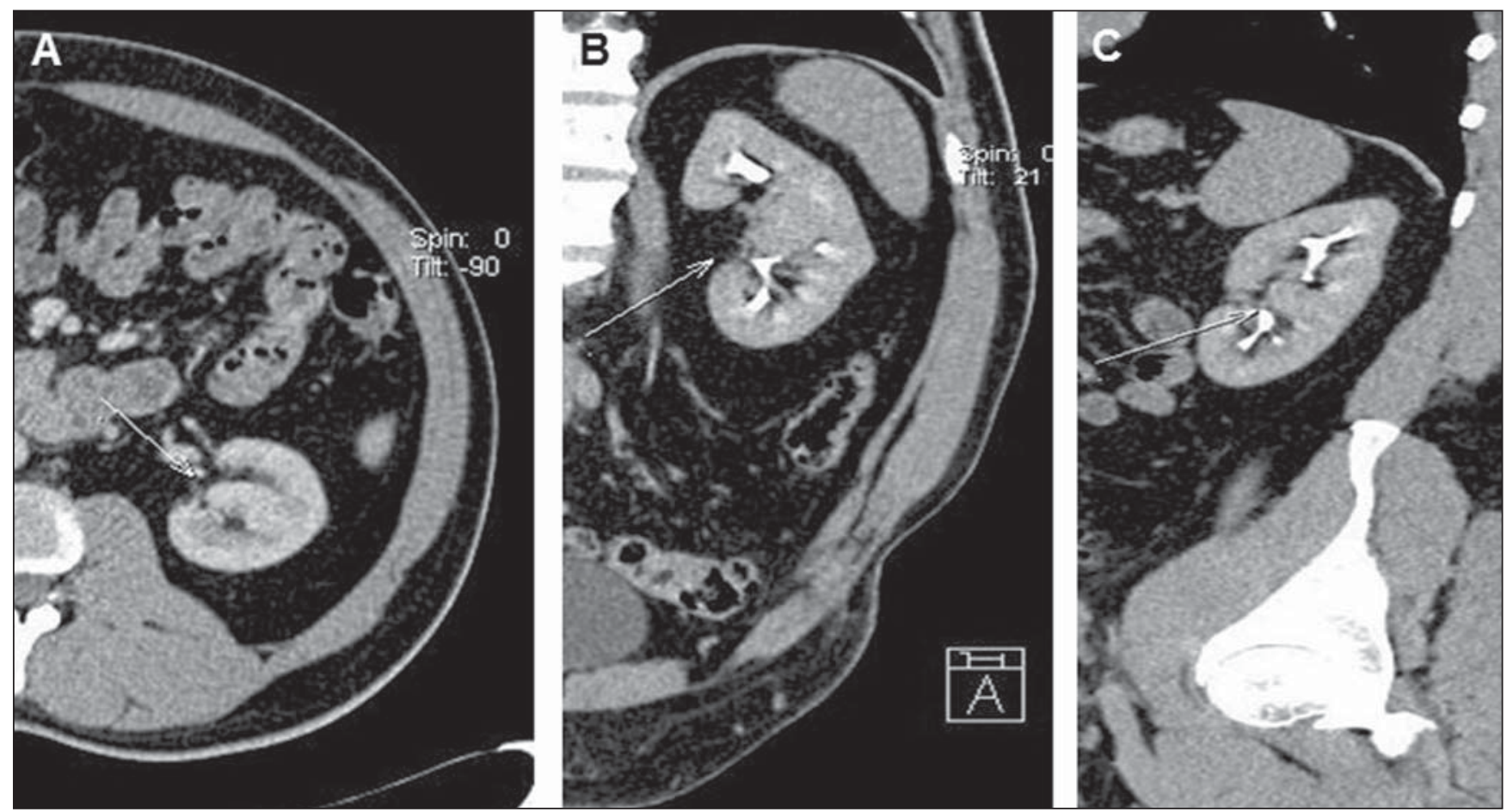

Figura 8. Hipertrofia do septo de Bertin. Imagens de TCMD axial (A), coronal (B) e sagital (C) demonstrando a hipertrofia dos septos. 

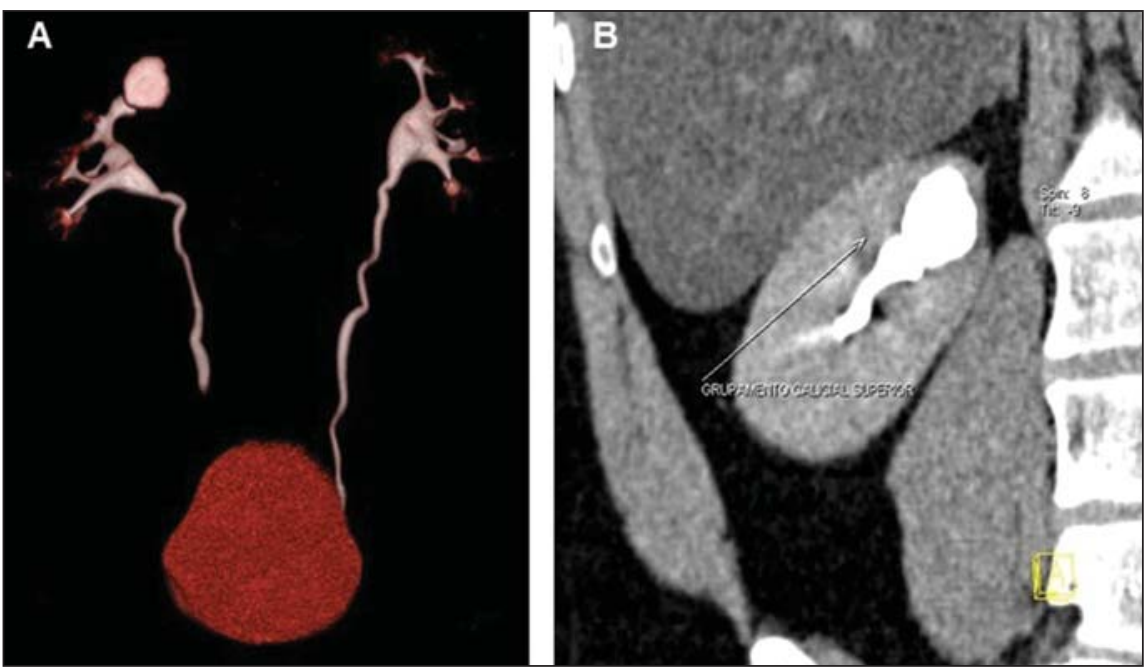

Figura 9. Divertículo pielocalicinal. Imagens em VRT (A) e coronal (B) do rim esquerdo evidenciando o divertículo calicinal em seu grupamento superior do rim direito.

\section{ANOMALIAS DA PELVE RENAL E DO URETER}

O sistema coletor renal é sede frequente de variações anatômicas com respeito a tamanho, forma, grau de ramificação e grau de rotação em relação ao hilo renal.

A estenose da junção ureteropélvica (JUP) (Figura 10) é a anomalia urinária mais comum na infância e mais frequente nas crianças do sexo masculino, normalmente sendo diagnosticada no primeiro ano de vida, mas que pode seguir não diagnosticada até a vida adulta, sendo nesta faixa etária mais comum em mulheres ${ }^{(\mathbf{1 , 2 2})}$. Nesta afecção há um estreitamento da JUP, geralmente à esquerda, que pode ser decorrente de lesão intrínseca muscular ou de descontinuidade funcional nesse segmento, que impede o esvaziamento adequado da pelve renal resultando em hidronefrose ${ }^{(15,22)}$.

A estenose também pode ser provocada por uma prega mucosa pieloureteral com comportamento valvular, ou ainda decorrente de compressão extrínseca por um vaso aberrante que comprime o infundíbulo da pelve renal e dificulta o seu esvaziamento $^{(15)}$. É uma das principais causas de dilatação do trato urinário (cerca de $35 \%$ a $40 \%$ dos casos) e sua origem não está totalmente esclarecida ${ }^{(\mathbf{1 8})}$.

Porém, a maior parte das anomalias da pelve renal e do ureter apresenta-se como duplicidade do sistema coletor, causa comum de assimetria de dimensões entre os rins durante a infância e que ocorre em $1 \%$ a $2 \%$ da população, sendo mais comum no sexo feminino ${ }^{(15,22,30)}$. Esta duplicação pode ser completa ou incompleta (Figura 11), com a forma unilateral mais comum que a bilateral, e frequentemente está associada a várias complicações ${ }^{(22,30)}$. O rim com duplo sistema coletor tem maior tamanho, sobretudo em seu eixo longitudinal, e maior volume do parênquima.

Na duplicidade completa (Figuras 11A e 11B) há dois sistemas coletores para um único rim e dois ureteres de um mesmo lado, que desembocam em orifícios separados. De acordo com a regra de WeigertMeyer (Figura 11B), o ureter que drena a parte superior passa pela parede da bexiga urinária para se inserir inferior e medialmente ao local normal de sua inserção. Com frequência, sua inserção é defeituosa, associada a ureterocele, e quando ectópica, pode desembocar na uretra posterior, na vagina ou na vulva. O ureter do segmento inferior insere-se próximo ao local de normalidade e está sujeito a refluxo vesicoureteral devido à distorção que sofre ao atravessar a parede da bexiga urinária associada a ureterocele ${ }^{(\mathbf{1 5 , 2 2})}$. À radiografia, a dilatação completa aparece como o sinal característico e bem conhecido, o dropping lily ${ }^{(\mathbf{2 1})}$.

Complicações da duplicação completa incluem infecções, refluxo vesicoureteral e obstrução da JUP. O refluxo no sistema coletor do segmento inferior pode produzir cicatrizes e deformidades deste segmento ${ }^{(22)}$.

Já na duplicidade incompleta (Figura 11C) há dois sistemas coletores e dois ureteres que se fundem, em qualquer nível, entre o rim e a bexiga (normalmente entre no terço inferior do trajeto), para originar um ureter único que desemboca normal-
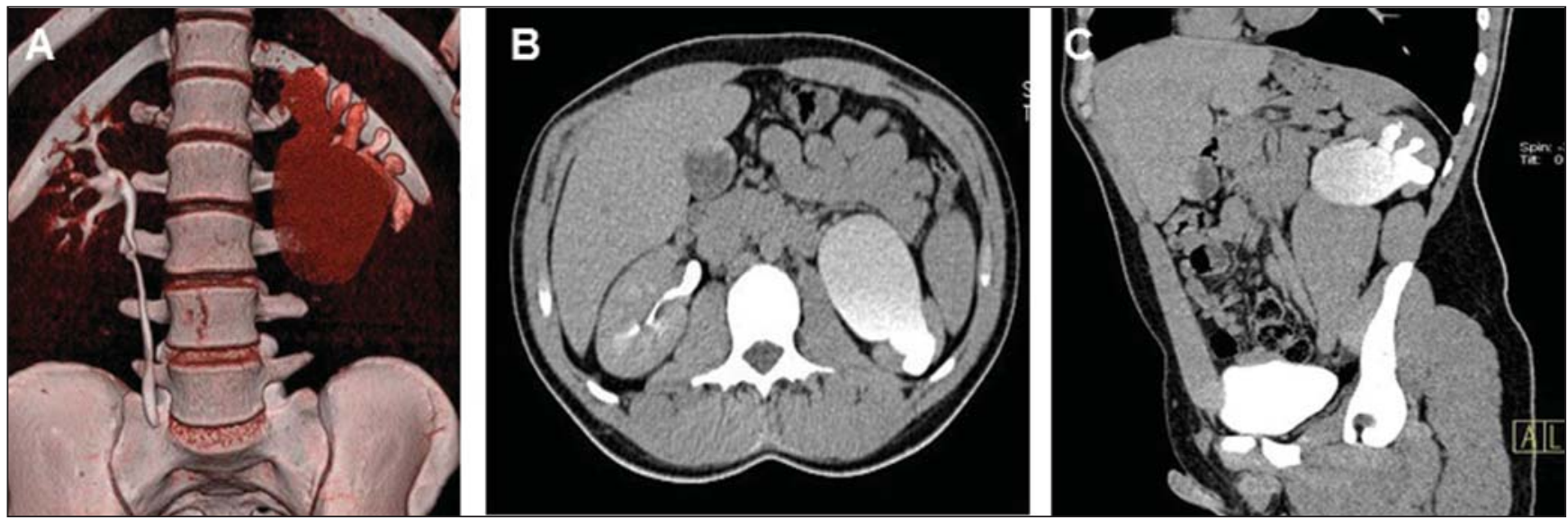

Figura 10. Estenose da junção pieloureteral (JUP). Imagens de TCMD em VRT (A), axial (B) e sagital oblíqua (C) revelando acentuada dilatação pielocalicinal à esquerda sem dilatação do ureter correspondente, caracterizando estenose da JUP. 

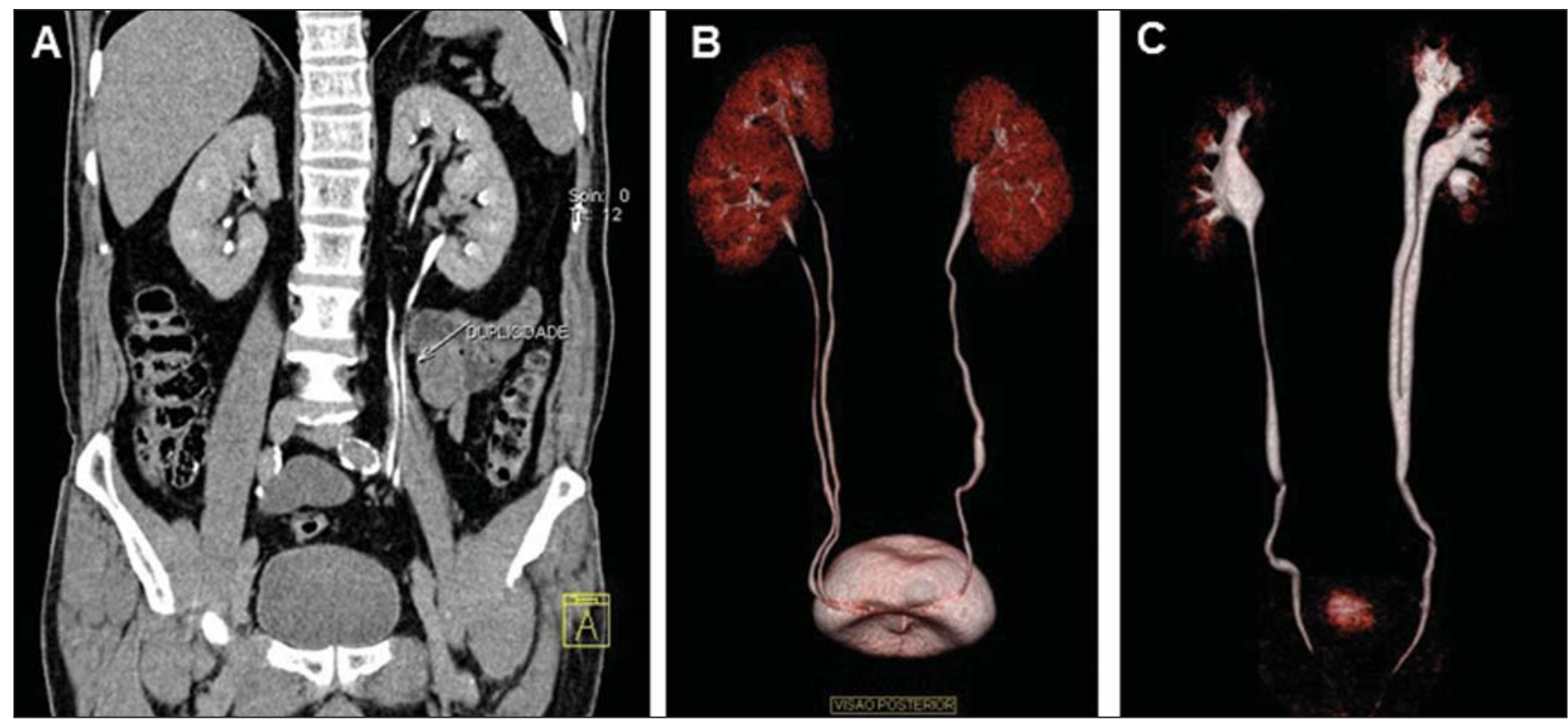

Figura 11. Duplicidade do sistema coletor. Imagens de TCMD coronal (A) e em VRT (B) mostrando duplicidade completa à esquerda. Notar, pela visão posterior em (B), a regra de Weigert-Meyer. A imagem em VRT (C) mostra duplicidade incompleta à esquerda, na qual se obsenva a união dos ureteres no terço médio inferior do trajeto ureteral. Notar a discreta dilatação pielocalicinal e dos dois ureteres acima da junção.

mente na base vesical. Quando a junção se dá em um nível acima da cúpula vesical, o ureter é chamado "em Y", e se ocorre ao nível do segmento intramural dos ureteres, tem-se ureter "em V",(15,22). Pode haver refluxo uretero-ureteral decorrente da assincronia entre o peristaltismo dos ureteres antes da confluência.

Outras anomalias que resultam da divisão do sistema coletor são as anomalias da pelve (Figura 12). Na pelve renal bífida (Figuras 12A e 12B) apenas a pelve renal está dividida, continuando a existir apenas uma JUP. É uma anomalia relativamente comum, podendo ocorrer em até $10 \%$ da população, não havendo associação com outras anomalias ${ }^{(22)}$. A posição da pelve renal também é bastante variável. Classifica-se a pelve como intrarrenal quando há tecido renal abundante ao seu redor. Em contrapartida, a pelve é extrarrenal (Figuras 12C e 12D), mais comum, quando está realmente fora do hilo, sendo este ocupado apenas pelos infundíbulos calicinais. Geralmente está associada a outras anomalias, como vícios de rotação ou de posição, podendo ocorrer estase e predisposição a infecções ${ }^{(\mathbf{2 2})}$.

\section{CONCLUSÃO}

Muitas das alterações morfológicas dos rins podem ser avaliadas por meio da US e
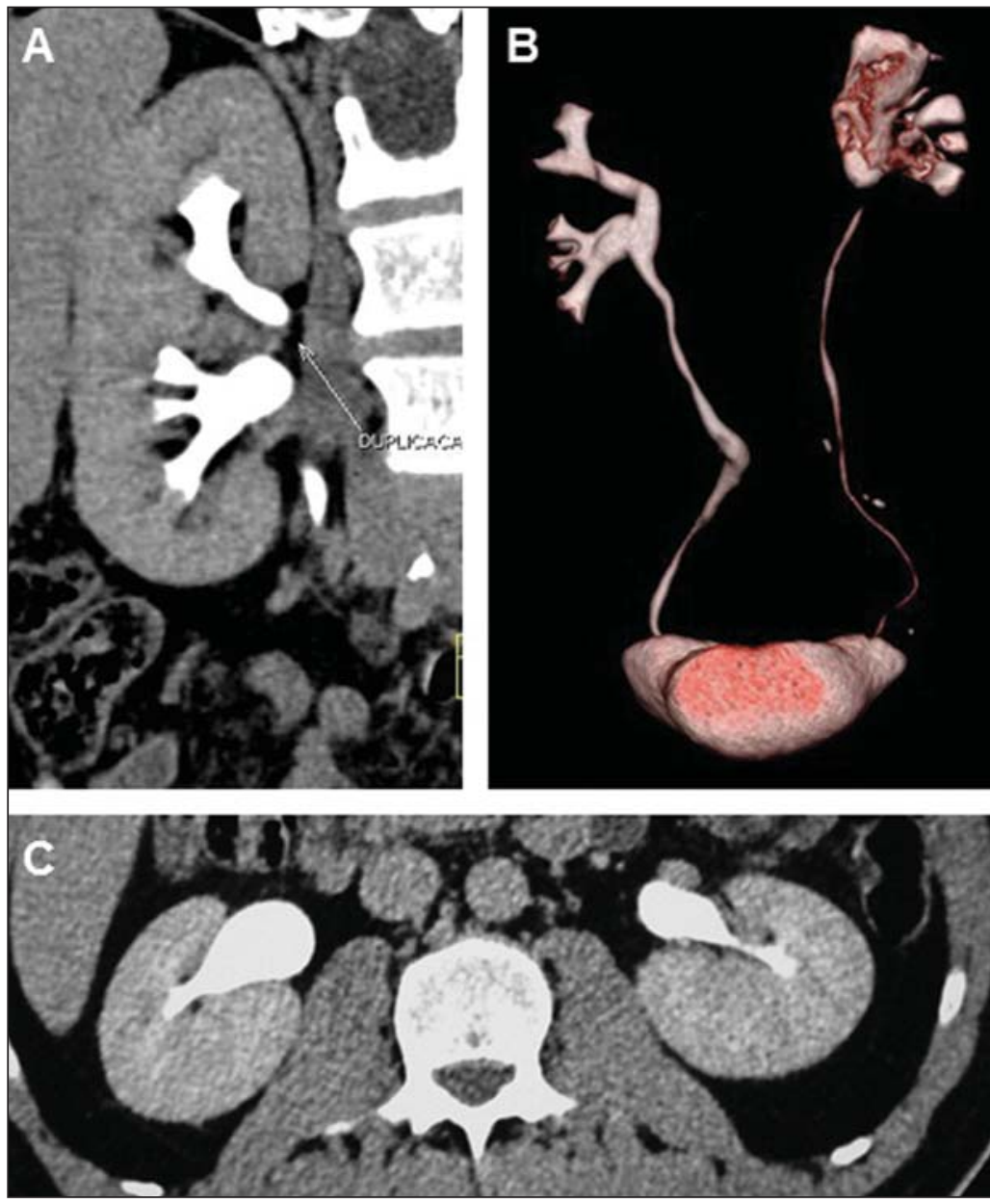

Figura 12. Anomalias da pelve renal. Imagens de TCMD coronal (A) e em VRT (B) identificando pelve renal bífida à direita. Imagem axial de TCMD $(\mathbf{C})$ demonstrando pelves extrarrenais. 
UE, porém a TC com os seus mais modernos avanços tecnológicos tem contribuído nos últimos anos para uma melhor caracterização das alterações morfológicas. A TC é fundamental no diagnóstico das anomalias congênitas, orientando melhor decisões terapêuticas clínicas e cirúrgicas e atuando como ferramenta essencial na identificação de complicações associadas. Os diversos recursos ainda permitem a avaliação renal mediante tamanho, posição e forma.

Neste trabalho são mostradas como as novas imagens das mesmas anomalias congênitas renais têm contribuído para o diagnóstico mais acurado e melhor avaliação das complicações nestes pacientes.

\section{REFERÊNCIAS}

1. Moore KL, Persaud TVN. Embriologia clínica. 6 ed. Rio de Janeiro, RJ: Guanabara Koogan; 2000.

2. Kumar V, Abbas AK, Fausto N. Robbins e Cotran - Patologia: bases patológicas das doenças. $7^{\mathrm{a}} \mathrm{ed}$. Rio de Janeiro, RJ: Elsevier; 2005.

3. Reidy KJ, Rosenblum ND. Cell and molecular biology of kidney development. Semin Nephrol. 2009;29:321-37

4. Sanna-Cherchi S, Ravani P, Corbani V, et al. Renal outcome in patients with congenital anomalies of the kidney and urinary tract. Kidney Int 2009;76:528-33.

5. Prando A, Prando D, Caserta NMG, et al. Urologia: diagnóstico por imagem. São Paulo, SP: Sarvier; 1997

6. Caoili EM, Cohan RH, Korobkin M, et al. Urinary tract abnormalities: initial experience with multi- detector row CT urography. Radiology. 2002;222: 353-60.

7. Nikken JJ, Krestin GP. MRI of the kidney - state of the art. Eur Radiol. 2007;17:2780-93.

8. Galvão Filho MM, D'Ippolito G, Hartmann LG, et al. O valor da tomografia computadorizada helicoidal sem contraste na avaliação de pacientes com dor no flanco. Radiol Bras. 2001;34:129-34.

9. Warshauer DM, McCarthy SM, Street L, et al. Detection of renal masses: sensitivities and specificities of excretory urography/linear tomography, US, and CT. Radiology. 1988;169:363-5.

10. Jamis-Dow CA, Choyke PL, Jennings SB, et al. Small $(<$ or $=3-\mathrm{cm})$ renal masses: detection with CT versus US and pathologic correlation. Radiology. 1996;198:785-8.

11. Smith RC, Verga M, McCarthy S, et al. Diagnosis of acute flank pain: value of unenhanced CT. AJR Am J Roentgenol. 1996;166:97-101.

12. Levine JA, Neitlich J, Verga M, et al. Ureteral calculi in patients with flank pain: correlation of plain radiography with unenhanced helical CT. Radiology. 1997;204:27-31

13. Kawashima A, Sandler CM, Ernst RD, et al. CT evaluation of renovascular disease. Radiographics. 2000;20:1321-40.

14. Barbaric ZL. Principles of genitourinary radiology. 2nd ed. New York, NY: Thieme; 1994.

15. Kim SH. Radiology illustrated - uroradiology. 2nd ed. Berlin: Springer-Verlag; 2012.

16. Hartshorne N, Shepard T, Barr M Jr. Compensatory renal growth in human fetuses with unilateral renal agenesis. Teratology. 1991;44:7-10.

17. Cho JY, Moon MH, Lee YH, et al. Measurement of compensatory hyperplasia of the contralateral kidney: usefulness for differential diagnosis of fetal unilateral empty renal fossa. Ultrasound Obstet Gynecol. 2009;34:515-20.

18. Fotter R. Pediatric uroradiology. 2nd ed. Berlin: Springer-Verlag; 2008.
19. Gun S, Ciantelli GL, Takahashi MAU, et al. Fusão renal completa em criança com infecção recorrente do trato urinário. Radiol Bras. 2012;45: 233-4.

20. Türkvatan A, Ölçer T, Cumhur T. Multidetector CT urography of renal fusion anomalies. Diagn Interv Radiol. 2009;15:127-34.

21. Dyer RB, Chen MY, Zagoria RJ. Classic signs in uroradiology. Radiographics. 2004;24 Suppl 1: S247-80.

22. Brant WE, Helms CA. Fundamentals of diagnostic radiology. 3rd ed. Philadelphia, PA: Lippincott Williams \& Wilkins; 2007.

23. Quaia E. Radiological imaging of the kidney. 1st ed. Berlin: Springer-Verlag; 2011.

24. Zhou Q, Cardoza JD, Barth R. Prenatal sonography of congenital renal malformations. AJR Am J Roentgenol. 1999;173:1371-6.

25. Bhatt S, MacLennan G, Dogra V. Renal pseudotumors. AJR Am J Roentgenol. 2007;188:13807

26. Lafortune M, Constantin A, Breton G, et al. Sonography of the hypertrophied column of Bertin. AJR Am J Roentgenol. 1986;146:53-6.

27. Rathaus V, Konen O, Werner M, et al. Pyelocalyceal diverticulum: the imaging spectrum with emphasis on the ultrasound features. Br J Radiol. 2001;74:595-601.

28. Abad PG, González IF, Peso AC, et al. Percutaneous treatment of stone-containing calyceal diverticulum. Arch Esp Urol. 2009;62:42-8.

29. Stunell H, McNeill G, Browne RF, et al. The imaging appearances of calyceal diverticula complicated by uroliathasis. Br J Radiol. 2010;83:88894

30. Fernbach SK, Feinstein KA, Spencer K, et al Ureteral duplication and its complications. Radiographics. 1997;17:109-27. 